\title{
Circulating Activin A Is a Surrogate for the Incidence of Diastolic Dysfunction and Heart Failure in Patients With Preserved Ejection Fraction
}

\author{
Yi-Lin Tsai, MD; Ruey-Hsing Chou, MD; Chin-Sung Kuo, MD; \\ Chun-Chin Chang, MD; Cheng-Hsueh Wu, MD; Po-Hsun Huang, MD, PhD; \\ Jaw-Wen Chen, MD; Shing-Jong Lin, MD, PhD
}

\begin{abstract}
Background: Diastolic dysfunction (DD) is a characteristic of heart failure with preserved ejection fraction (HFpEF), which is thought to be caused by cardiac hypertrophy or fibrosis. Activin A is involved in the inflammatory response and myocardial fibrosis, but the relationship between the activin $A$ level and $D D$ remains unclear.

Methods and Results: A total of 209 patients with stable angina were enrolled. Serum activin A levels were assessed, and echocardiography and cross-sectional analysis were performed. Among the subjects (65\% male; mean age, $70 \pm 13$ years), 84 (40\%) subjects had DD. The subjects were divided into tertiles based on activin A levels. Patients in the high activin A group had enhanced left ventricular mass indexes, medial E/e' ratios, left atrial diameter, and right ventricular systolic pressure compared with those in the lower activin A groups (all $P<0.001)$. Prevalence of DD ( $P=0.001), H F p E F$ at enrollment $(P=0.007)$, and the composite endpoints including new-onset heart failure (HF) or death within 3 years $(P<0.001)$ correlated positively with high activin $A$ levels. After adjusting for confounding factors, high activin A levels remained significantly associated with $D D(P=0.036)$ and the composite endpoints $(\mathrm{P}=0.012)$.
\end{abstract}

Conclusions: Enhanced serum activin A levels were associated with the incidence of DD and development of HF.

Key Words: Activin A; Diastolic dysfunction; Heart failure

$\mathbf{H}$ eart failure (HF) is a severe clinical syndrome that has become a major health problem worldwide. Approximately half of the patients with HF have a preserved left ventricular ejection fraction (LVEF), and the prevalence of HF with preserved ejection fraction (HFpEF) continues to grow because of the rapid increase in aging of populations. Diastolic dysfunction (DD) is defined broadly as impaired relaxation and increased stiffness of the LV, which affect blood filling during diastole. Emerging evidence suggests that patients with DD are at significantly greater risk of symptomatic $\mathrm{HF}$ and that this condition is predictive of all-cause death., ${ }^{1,2}$ Although DD is prevalent in the clinical setting, its pathophysiology and clinical relevance are not fully understood. Epidemiological evidence suggests the existence of a latent phase between DD and

\section{Editorial p1443}

progression to symptomatic HF. ${ }^{1,3}$

Many mechanisms have been proposed to clarify the development of DD. Cardiac fibrosis and myocardial remodeling are known to play especially critical roles. ${ }^{3,4}$ Activin A, a member of the transforming growth factor- $\beta$ (TGF- $\beta$ ) cytokine superfamily, was identified as an inducer of follicle-stimulating hormone release during the 1980s. ${ }^{5}$ Previous reports have revealed that activin $\mathrm{A}$ is secreted by different types of cell and acts as a multifunctional cytokine; it has also been shown to play pivotal roles in immune modulation and inflammatory responses. ${ }^{5-7}$ Emerging evidence suggests that activin A has effects on the cardiovascular

Received July 24, 2018; revised manuscript received March 24, 2019; accepted April 2, 2019; J-STAGE Advance Publication released online May 14, 2019 Time for primary review: 28 days

Division of Cardiology, Department of Medicine, Taipei Veterans General Hospital, Taipei (Y.-L.T., R.-H.C., C.-C.C., C.-H.W., P.-H.H., J.-W.C., S.-J.L.); Cardiovascular Research Center (Y.-L.T., R.-H.C., C.-S.K., C.-C.C., C.-H.W., P.-H.H., J.-W.C., S.-J.L.), Institute of Clinical Medicine (Y.-L.T., R.-H.C., C.-S.K., C.-C.C., P.-H.H., S.-J.L.), National Yang-Ming University, Taipei; Division of Endocrinology and Metabolism, Department of Medicine (C.-S.K.), Department of Critical Care Medicine (R.-H.C., C.-H.W., P.-H.H.), Division of Clinical Research, Department of Medical Research (J.-W.C.), Taipei Veterans General Hospital, Taipei; Institute of Pharmacology, National Yang-Ming University, Taipei (J.-W.C.); and Taipei Medical University, Taipei (S.-J.L.), Taiwan

Mailing address: Po-Hsun Huang, Professor, MD, PhD, Department of Critical Care Medicine, Taipei Veterans General Hospital, 112 , No. 201, Sec. 2, Shih-Pai Road, Taipei, Taiwan. E-mail: huangbs@vghtpe.gov.tw or Chin-Sung Kuo, MD, Department of Medicine, Taipei Veterans General Hospital, 112, No. 201, Sec. 2, Shih-Pai Road, Taipei, Taiwan. E-mail: cskuo241@yahoo.com.tw

ISSN-1346-9843 All rights are reserved to the Japanese Circulation Society. For permissions, please e-mail: cj@j-circ.or.jp 
system. Elevated levels of activin A have been identified in patients with HF, pulmonary hypertension, systemic hypertension, and atherosclerosis. ${ }^{\mathbf{6}, \mathbf{8}-13}$ Activin A stimulates fibroblast proliferation and differentiation into myofibroblasts in response to tissue inflammation, which is an essential step in cardiac remodeling. Moreover, activin A induces the expression of TGF- $\beta$ and multiple regulators, such as connective tissue growth factor, tissue inhibitor of metalloproteinase-1, plasminogen activator inhibitor 1 , and type 1 collagen, potentiating tissue fibrosis. ${ }^{5}$ Production of activin $\mathrm{A}$ in various tissues is driven by other fibrosis regulators, such as tumor necrosis factor- $\alpha$ and interleukin-13, which suggests that activin A is a critical cytokine in myocardial fibrosis. ${ }^{5}$ However, the relationship between the serum activin A level and DD in human subjects remains unclear. Therefore, we designed this study to investigate the correlation between the serum activin A level and diastolic function, assessed by echocardiography.

\section{Methods}

\section{Study Population}

During February 2010 to December 2014, 515 patients with symptoms of angina were screened at Taipei Veterans General Hospital. Patients older than 18 years who were eligible for the study underwent routine echocardiographic screening. Patients with LV systolic dysfunction (EF <50\%) and those with severe valvular heart disease were excluded. All participants provided written informed consent, and the study was approved by the Research Ethics Committee of Taipei Veterans General Hospital.

\section{Demography and Laboratory Examinations}

Each patient's chart was reviewed in detail to collect past medical history. If the past record was not available at the study hospital, the patient was asked to complete a questionnaire to provide medical history information including chronic disease and daily medications. All residents underwent anthropometric measurement by research nurses, including height, body weight, blood pressure (BP) and waist circumference. The body mass index (BMI) was calculated as weight (in kilograms) divided by height (in meters) squared.

Blood samples were obtained from all patients after overnight fasting of at least $10 \mathrm{~h}$. The serum creatinine level was measured using an automatic analyzer (AVDIA 1800; Siemens, Malvern, PA, USA). The estimated glomerular filtration rate (eGFR) was calculated using the Chronic Kidney Disease Epidemiology Collaboration equation. ${ }^{14}$ The serum levels of N-terminal pro-B-type natriuretic peptide (NT-proBNP) were determined by commercial enzymelinked immunosorbent assay (ELECSYS proBNPRoche Diagnostics, Germany) using an ELECSYS2010 instrument. Serum concentrations of activin A (R\&D Quantikine ELISA Human Activin A immunoassay, USA) and highsensitivity C-reactive protein (hs-CRP) (ELISA, Beckman Coulter Inc., Brea, CA, USA) were also measured by immunoassays. The participants were stratified into tertiles based on the serum level of activin A for further analysis: low $(<305 \mathrm{pg} / \mathrm{mL}), \mathrm{n}=70$; medium $(305-435 \mathrm{pg} / \mathrm{mL}), \mathrm{n}=69$; high $(>435 \mathrm{pg} / \mathrm{mL}), \mathrm{n}=70$.

\section{Echocardiography and Definition of DD}

The participants underwent echocardiography in 3 consecutive months after enrollment. Experienced technicians per- formed the imaging using an IE33 device (Philips Healthcare, Amstelplein 2, 1096 BC Amsterdam, The Netherlands). The LV end-diastolic dimension (LVDd), interventricular septal thickness (IVST), and posterior wall thickness (PWT) were assessed in the parasternal long-axis view at end diastole. The left atrial dimension (LAD) was measured at end systole. We calculated the LV mass (LVM) using the formula proposed by Devereux et $\mathrm{al}^{15}$ with adjustment: $0.8 \times 1.04 \times\left[\left(\mathrm{LVDd}^{2} \mathrm{IVST}+\mathrm{PWT}\right)^{3}-\mathrm{LVDd}^{3}\right]+0.6$. The LVM index (LVMI) was calculated as the ratio of the LVM to the body surface area. The LVEF was evaluated using Simpson's method, with LV end-diastolic and end-systolic volumes acquired in the apical 4-chamber views. The transmitral inflow was measured by pulsed-wave blood flow Doppler at the mitral valve leaflet tips in the apical 4-chamber view. The peak velocities of early filling (E), atrial filling (A), and the deceleration time of the $\mathrm{E}$ wave were obtained. The early peak diastolic mitral annulus velocity (e') was determined by pulsed-wave tissue Doppler at the LV septum. Right ventricular systolic pressure (RVSP) was estimated using the maximal velocity of the tricuspid regurgitation jet, measured by continuous-wave blood flow Doppler. LV hypertrophy was defined as LVMI $>118 \mathrm{~g} / \mathrm{m}^{2}$ in men and LVMI $>108 \mathrm{~g} / \mathrm{m}^{2}$ in women. ${ }^{16}$ Based on previous reports, ${ }^{17-19}$ the diagnosis of cardiac diastolic dysfunction was made when one of the following echocardiographic criteria was met: (1) septal e' $<8$ and E/e' $\geq 15$, (2) septal e' $<8$ and $\mathrm{E} / \mathrm{e}^{\prime} \geq 8$ and $\mathrm{E} / \mathrm{A}<0.5$ and deceleration time of $\mathrm{E}$ wave $\geq 280 \mathrm{~ms}$, or (3) septal e' $<8$ and E/e' $\geq 8$ and E/A $<0.5$ and presence of LV hypertrophy.

\section{Diagnosis and Follow-up of HF}

Diagnosis of HF was based on the definition and classifications of the 2013 AHA/ACC guideline for the management of HF ${ }^{20}$ After completion of echocardiography and exclusion of participants with impaired LV systolic function, all participants were examined in detail to determine if they met the diagnosis of HFpEF. All patients were followed up for 3 years by regular outpatient clinic visits every 3-6 months. The occurrence of all-cause death or new-onset $\mathrm{HF}$ during the follow-up period was recorded as the composite endpoint.

\section{Statistical Analysis}

Categorical variables are expressed as percentages and descriptive continuous variables are expressed as mean \pm standard deviation. The Kruskal-Wallis test was used to compare continuous variables and the $\chi^{2}$ test was used to compare categorical data. The contribution of each variable was analyzed using the Spearmen correlation coefficient. Logistic regression was performed to analyze the correlation between activin A level, DD, and the composite endpoint. Potential confounding factors were carefully adjusted in the multivariable regression analysis. All statistical analyses were performed with SPSS software (SPSS, version 24.0.0.0, IBM Corporation, Armonk, NY, USA). Two-tailed P-values $<0.05$ were considered to be statistically significant.

\section{Results}

\section{General Participant Characteristics}

In total, 209 patients (65.6\% male; mean age, $70 \pm 13$ years) were enrolled in the present study. Table 1 summarizes their demographic characteristics. Compared with those in 


\begin{tabular}{|c|c|c|c|c|c|c|}
\hline & $\begin{array}{c}\text { Total } \\
{[n=209]}\end{array}$ & $\begin{array}{l}\text { Tertile I } \\
{[n=70]} \\
(<305)\end{array}$ & $\begin{array}{c}\text { Tertile II } \\
{[n=69]} \\
(\geq 305, \leq 435)\end{array}$ & $\begin{array}{c}\text { Tertile III } \\
{[n=70]} \\
(>435)\end{array}$ & $P$ value & $\begin{array}{l}P \text { for } \\
\text { trend }\end{array}$ \\
\hline \multicolumn{7}{|l|}{ Clinical characteristics } \\
\hline Age (years) & $70 \pm 13$ & $64 \pm 13$ & $74 \pm 10$ & $72 \pm 14$ & $<0.001$ & $<0.001$ \\
\hline Male (\%) & $137(65.6)$ & $45(64.3)$ & $46(66.7)$ & $46(65.7)$ & 0.957 & 0.859 \\
\hline BMI $\left(k g / m^{2}\right)$ & $25.8 \pm 3.8$ & $26.1 \pm 4.2$ & $25.7 \pm 3.4$ & $25.5 \pm 3.9$ & 0.696 & 0.397 \\
\hline Smoking (\%) & $101(37.1)$ & $20(24.1)$ & 35 (39.3) & $46(46.0)$ & $<0.008$ & $<0.003$ \\
\hline HTN (\%) & $160(76.6)$ & $45(64.3)$ & $56(81.2)$ & $59(84.3)$ & 0.011 & 0.005 \\
\hline $\mathrm{SBP}(\mathrm{mmHg})$ & $129.9 \pm 13.9$ & $124.9 \pm 11.2$ & $129.8 \pm 12.4$ & $135.1 \pm 16.0$ & $<0.001$ & $<0.001$ \\
\hline $\mathrm{DBP}(\mathrm{mmHg})$ & $73.4 \pm 11.4$ & $73.5 \pm 10.0$ & $72.5 \pm 8.8$ & $74.2 \pm 14.5$ & 0.651 & 0.716 \\
\hline $\mathrm{PP}(\mathrm{mmHg})$ & $56.5 \pm 14.3$ & $51.3 \pm 12.2$ & $57.3 \pm 12.1$ & $60.9 \pm 16.8$ & $<0.001$ & $<0.001$ \\
\hline DM (\%) & $71(34.0)$ & $18(25.7)$ & $27(39.1)$ & $26(37.1)$ & 0.196 & 0.154 \\
\hline $\mathrm{AF}(\%)$ & $35(16.7)$ & $8(11.4)$ & $10(14.5)$ & $17(24.3)$ & 0.104 & 0.042 \\
\hline CKD (\%) & $27(12.7)$ & $1(1.4)$ & $2(2.9)$ & $24(34.3)$ & $<0.001$ & $<0.001$ \\
\hline eGFR $\left(\mathrm{mL} / \mathrm{min} / 1.73 \mathrm{~m}^{2}\right)$ & $62.1 \pm 25.0$ & $74.9 \pm 18.2$ & $62.0 \pm 17.6$ & $49.6 \pm 30.4$ & $<0.001$ & $<0.001$ \\
\hline hs-CRP (mg/L) & $0.7 \pm 1.5$ & $0.2 \pm 0.3$ & $0.5 \pm 1.4$ & $1.5 \pm 2.1$ & $<0.001$ & $<0.001$ \\
\hline NT-proBNP & $3,287.1 \pm 10,390.2$ & $756.3 \pm 3,348.0$ & $1,706.1 \pm 4,271.2$ & $7,376.4 \pm 16,440.4$ & $<0.001$ & $<0.001$ \\
\hline \multicolumn{7}{|l|}{ Antihypertension medication } \\
\hline ACEI/ARB (\%) & $87(32.0)$ & $22(26.5)$ & $31(34.8)$ & $34(34.0)$ & 0.435 & 0.298 \\
\hline$\beta$-blocker (\%) & $74(27.2)$ & $18(21.7)$ & $23(25.8)$ & $33(33.0)$ & 0.217 & 0.084 \\
\hline CCB $(\%)$ & $69(25.4)$ & $12(14.5)$ & $29(32.6)$ & $28(28.0)$ & 0.018 & 0.047 \\
\hline Diuretics (\%) & $48(17.6)$ & $4(4.8)$ & $17(19.1)$ & $27(27.0)$ & $<0.001$ & $<0.001$ \\
\hline \multicolumn{7}{|l|}{ Echocardiography } \\
\hline LVEF (\%) & $0.59 \pm 0.06$ & $0.59 \pm 0.07$ & $0.58 \pm 0.06$ & $0.54 \pm 0.10$ & 0.001 & $<0.001$ \\
\hline LVMI & $109.1 \pm 47.2$ & $94.3 \pm 29.3$ & $107.1 \pm 47.4$ & $125.7 \pm 56.0$ & $<0.001$ & $<0.001$ \\
\hline E_A ratio & $0.87 \pm 0.3$ & $0.94 \pm 0.3$ & $0.81 \pm 0.4$ & $0.88 \pm 0.4$ & 0.055 & 0.244 \\
\hline Medial E/e' & $15.4 \pm 8.7$ & $11.6 \pm 4.1$ & $15.4 \pm 7.7$ & $19.4 \pm 11.1$ & $<0.001$ & $<0.001$ \\
\hline Septal e' & $6.08 \pm 2.22$ & $6.98 \pm 2.10$ & $5.52 \pm 1.92$ & $5.67 \pm 2.33$ & $<0.001$ & $<0.001$ \\
\hline $\mathrm{LAD}(\mathrm{mm})$ & $39.8 \pm 7.3$ & $36.8 \pm 5.7$ & $38.6 \pm 5.7$ & $43.9 \pm 8.4$ & $<0.001$ & $<0.001$ \\
\hline RVSP (mmHg) & $34.0 \pm 12.6$ & $29.3 \pm 6.6$ & $32.6 \pm 11.5$ & $39.2 \pm 15.5$ & $<0.001$ & $<0.001$ \\
\hline \multicolumn{7}{|l|}{ Clinical outcome } \\
\hline DD (\%) & $84(40.2)$ & $17(24.3)$ & $28(40.6)$ & $39(55.7)$ & 0.001 & $<0.001$ \\
\hline HFpEF at enrollment (\%) & $20(9.6)$ & $3(4.3)$ & $4(5.8)$ & $13(18.6)$ & 0.007 & 0.004 \\
\hline New HF in 3 years (\%) & $22(10.5)$ & $2(2.9)$ & $8(11.6)$ & $12(17.1)$ & 0.021 & 0.012 \\
\hline Death in 3 years (\%) & $12(5.7)$ & $0(0.0)$ & $4(5.8)$ & $8(11.4)$ & 0.015 & 0.004 \\
\hline Composite endpoint ${ }^{\star}(\%)$ & $31(14.8)$ & $2(2.9)$ & $10(14.5)$ & $19(27.1)$ & $<0.001$ & $<0.001$ \\
\hline
\end{tabular}

Values are mean \pm standard deviation or $n(\%)$. ${ }^{*}$ Composite endpoint=new-onset heart failure or death in 3 years. ACEl, angiotensin-converting enzyme inhibitor; AF, atrial fibrillation; ARB, angiotensin II receptor blocker; BMI, body mass index; CCB, calcium-channel blocker; CKD, chronic kidney disease; DBP, diastolic blood pressure; DD, diastolic dysfunction; DM, diabetes mellitus; eGFR, estimated glomerular filtration rate; HFpEF, heart failure with preserved EF; hs-CRP, high-sensitivity C-reactive protein; LAD, left atrial diameter; LVEF, left ventricular ejection fraction; LVMI, left ventricular mass index; NT-pro BNP, N-terminal pro-B-type natriuretic peptide; PP, pulse pressure; RVSP, right ventricular systolic pressure; SBP, systolic blood pressure.

the medium and low activin A groups, patients in the high activin A group were older (Tertile III: 71.8 \pm 13.7 , Tertile II: $74.3 \pm 9.2$, Tertile I: $63.5 \pm 13.1$, with a higher percentage of smokers (Tertile III: 46 [46.0\%], Tertile II: 35 [39.3\%], Tertile I: 20 [24.1\%]), hypertension (Tertile III: 59 [84.3\%], Tertile II: 56 [81.2\%], Tertile I: 45 [64.3\%]), chronic kidney disease (Tertile III: 24 [34.3\%], Tertile II: 2 [2.9\%], Tertile I: 1 [1.4\%]), and atrial fibrillation (Tertile III: 17 [24.3\%], Tertile II: 10 [14.5\%], Tertile I: 8 [11.4\%]).

The hs-CRP (Tertile III: $1.5 \pm 2.1$, Tertile II: $0.5 \pm 1.4$, Tertile I: $0.2 \pm 0.3$ ) and NT-proBNP levels (Tertile III: 7,376.4 $\pm 16,440.4$, Tertile II: $1,706.1 \pm 4,271.2$, Tertile I: $756.3 \pm 3,348.0$ ) were significantly higher in patients in the high activin A group. Usage of calcium-channel blockers (Tertile III: 28 [28.0\%], Tertile II: 29 [32.6\%], Tertile I: 12 [14.5\%]) and diuretic agents (Tertile III: 27 [27.0\%], Tertile II: 17 [19.1\%], Tertile I: $4[4.8 \%])$ for systemic hypertension control was more common in patients in the high and medium activin A groups.

The activin A level correlated inversely with the eGFR (Tertile III: $49.6 \pm 30.4$, Tertile II: $62.0 \pm 17.6$, Tertile I: 74.9 \pm 18.2 ). The BMI and prevalence of type 2 diabetes mellitus did not differ among groups.

\section{Associations Among Activin A Level, Diastolic Function, and Composite Endpoint}

Analysis of the echocardiographic parameters showed that patients in the high activin A group had significantly increased LVMI, medial E/e' values, LAD, and RVSP compared with those in the low and medium activin A groups (all $\mathrm{P}<0.001)$. The prevalence of cardiac diastolic dysfunc- 
Table 2. Correlation Coefficients for DD, Activin A Level, and Other Indicators of DD

\begin{tabular}{cccccccccc} 
Variable & Age & SBP & PP & eGFR & NT-proBNP & LVMI & LAD & RVSP & hs-CRP \\
Activin A & $0.310^{\star \star}$ & $0.297^{\star *}$ & $0.248^{\star *}$ & $-0.389^{\star \star}$ & $0.525^{\star \star}$ & $0.323^{\star *}$ & $0.386^{\star \star}$ & $0.263^{\star \star}$ & $0.380^{\star \star}$ \\
\hline
\end{tabular}

${ }^{* *}$ Correlation is significant at the 0.01 level (two-tailed). Abbreviations as in Table 1.
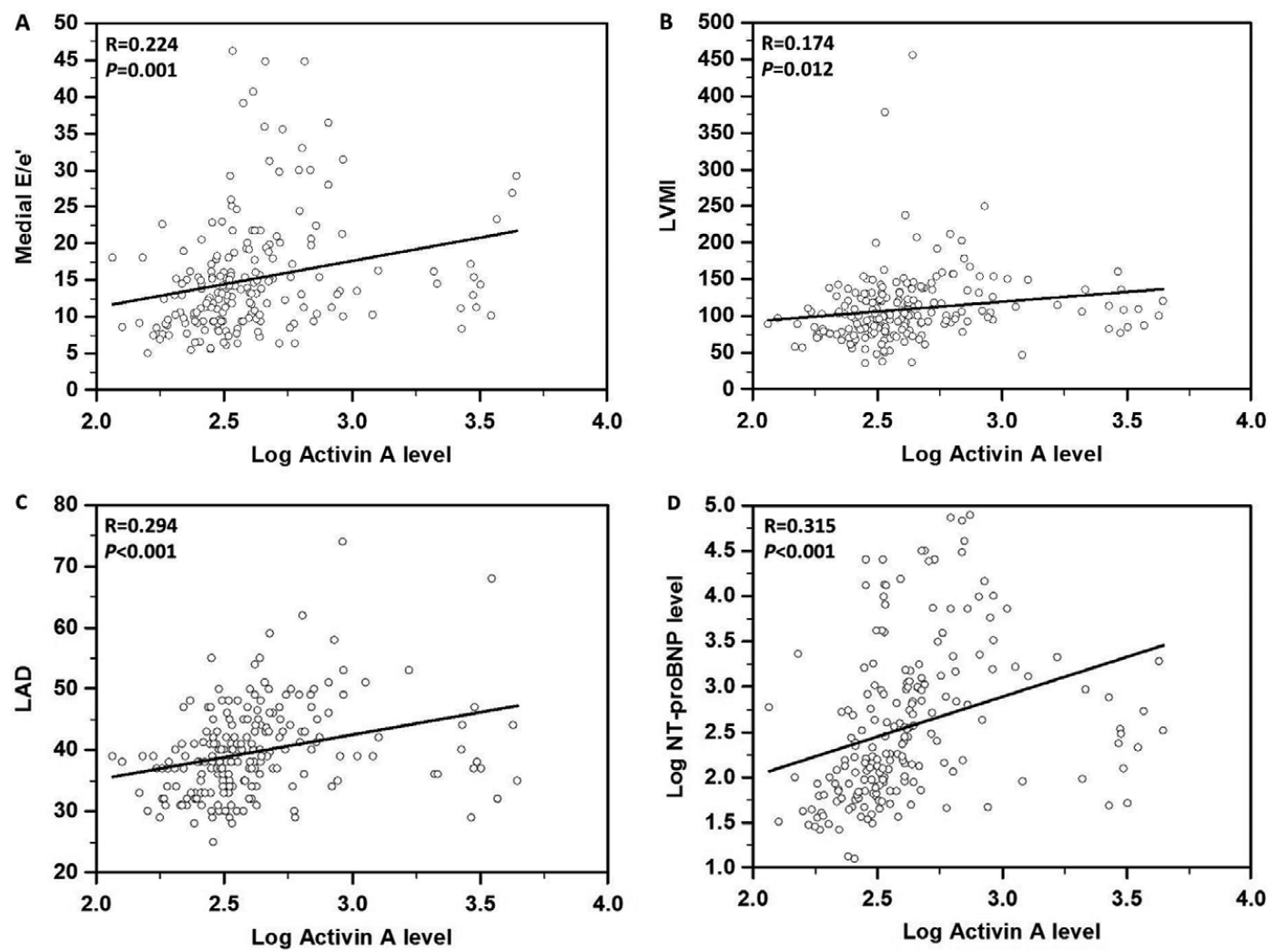

Figure. (A-D) Scatter plots of correlation of the serum level of activin A with medial E/e', LVMI, LAD, and log NT-proBNP. LAD, left atrial diameter; LVMI, left ventricular mass index; NT-proBNP, N-terminal pro-B-type natriuretic peptide.

\begin{tabular}{|lccc|}
\hline \multicolumn{3}{|l}{ Table 3. Logistic Regression Analysis for DD } \\
Dependent variable & $\begin{array}{c}\text { Odds } \\
\text { ratio }\end{array}$ & $\begin{array}{c}\text { Confidence } \\
\text { interval }\end{array}$ & P value \\
Model 1 & & - & - \\
$\quad$ Activin A: tertile I & Ref. & - & - \\
Activin A: tertile II & 1.55 & $0.72-3.35$ & 0.263 \\
Activin A: tertile III & 3.15 & $1.49-6.65$ & 0.003 \\
Model 2 & & & \\
Activin A: tertile II & 1.83 & $0.81-4.10$ & 0.145 \\
$\quad$ Activin A: tertile III & 3.53 & $1.48-8.41$ & 0.004 \\
Model 3 & & & \\
Activin A: tertile II & 1.57 & $0.68-3.60$ & 0.288 \\
Activin A: tertile III & 3.37 & $1.40-8.13$ & 0.007 \\
\hline
\end{tabular}

Model 1: adjusted for age and activin A. Model 2: adjusted for age, SBP, PP, smoking, eGFR, and activin A. Model 3: adjusted for age, SBP, PP, smoking, eGFR, LVEF, CCB, diuretics and activin A. Abbreviations as in Table 1.

\begin{tabular}{|c|c|c|c|}
\hline \multicolumn{4}{|c|}{$\begin{array}{l}\text { Table 4. Logistic Regression Analysis for Composite } \\
\text { Endpoints* }\end{array}$} \\
\hline Dependent variable & $\begin{array}{l}\text { Odds } \\
\text { ratio }\end{array}$ & $\begin{array}{l}\text { Confidence } \\
\text { interval }\end{array}$ & $P$ value \\
\hline \multicolumn{4}{|l|}{ Model 1} \\
\hline Activin A: tertile I & Ref. & - & - \\
\hline Activin A: tertile II & 3.62 & $0.74-17.71$ & 0.112 \\
\hline Activin A: tertile III & 8.78 & $1.90-40.58$ & 0.005 \\
\hline \multicolumn{4}{|l|}{ Model 2} \\
\hline Activin A: tertile II & 3.99 & $0.81-19.81$ & 0.090 \\
\hline Activin A: tertile III & 8.45 & $1.68-42.56$ & 0.010 \\
\hline \multicolumn{4}{|l|}{ Model 3} \\
\hline Activin A: tertile II & 4.14 & 0.83-20.79 & 0.084 \\
\hline Activin A: tertile III & 8.16 & $1.59-42.01$ & 0.012 \\
\hline
\end{tabular}

Model 1: adjusted for age and activin A. Model 2: adjusted for age, SBP, PP, smoking, eGFR, and activin A. Model 3: adjusted for age, SBP, PP, smoking, eGFR, LVEF, CCB, diuretics and activin A. *Composite endpoint: new-onset heart failure or death in 3 years. Abbreviations as in Table 1. 
tion $(\mathrm{P}=0.001), \mathrm{HFpEF}$ at enrollment $(\mathrm{P}=0.007)$, and the composite endpoint (death and new-onset HF $(\mathrm{P}<0.001)$ ) were associated positively with the activin A concentration.

Table 2 lists the correlation coefficients for the study variables, including echocardiographic parameters associated with DD and the serum level of activin A. Age, systolic BP (SBP), pulse pressure (PP), eGFR, NT-proBNP level, hs-CRP level, LVMI, LAD, and RVSP were significantly correlated with DD and the serum activin A levels. Scatter plots of the correlation between serum levels of activin A and medial E/e' values, LVMI, LADs, NT-proBNP are presented in Figure.

Using the low activin A tertile as the reference, the high activin A tertile was significantly associated with DD even after adjustment of potential confounding factors, including age $(\mathrm{P}=0.003$, model 1$), \mathrm{SBP}, \mathrm{PP}$, smoking, eGFR $(\mathrm{P}=0.004$, model 2$), \mathrm{LVEF}$, and usage of CCBs and diuretics $(\mathrm{P}=0.007$, model 3; Table 3).

Table 4 shows the logistic regression analysis of the composite endpoint. Compared with the low activin A tertile, the high activin A tertile was significantly associated with the composite endpoint even after adjusting age $(\mathrm{P}=0.005$, model 1), SBP, PP, smoking, eGFR ( $\mathrm{P}=0.010$, model 2$)$, LVEF, and usage of CCBs and diuretics $(\mathrm{P}=0.012$, model 3; Table 3).

\section{Discussion}

To our knowledge, this is the first study to demonstrate a positive association between the serum activin A concentrations and DD. Subjects with elevated levels of circulating activin A had significantly enhanced LVMI, LAD, and RVSP, and echocardiography showed DD in these subjects. Moreover, the prevalence of HFpEF and development of HF over 3 consecutive years were also significantly higher in patients with high activin A. Although the effects of activin A on inflammation and fibrotic processes in the cardiovascular system have been emphasized, ${ }^{\mathbf{5}}$ evidence connecting in vitro processes with clinical pathophysiology is lacking. Our findings provide clear evidence that the serum activin A level is associated with LV DD, which suggests a potential role of activin A in the development of DD. However, further studies are needed to evaluate the causal relationships and establish the clinical significance of these findings.

$\mathrm{HF}$ is a severe clinical syndrome that continues to be a major health problem worldwide. Epidemiological studies have shown that HFpEF is as prevalent as HF with reduced EF (HFrEF). Although a limited amount of research has focused on pure DD, increasing evidence suggests that patients with DD are at significantly greater risk of symptomatic $\mathrm{HF}$ and that this condition is predictive of all-cause death. ${ }^{1}$ Given the rapidly growing incidence of $\mathrm{HF}$, the molecular and clinical meanings for DD must be established to enable greater understanding and the development of effective treatment options.

Activin A, a homodimer of activin $\beta$ A subunits, belongs to the TGF- $\beta$ superfamily. Although originally described as an inducer of follicle-stimulating hormone release, activin $\mathrm{A}$ has been recognized as a multifunctional cytokine expressed in a wide range of tissues and cells, with roles in the regulation of wound repair, cell differentiation, apoptosis, and inflammation. In previous studies, elevated serum levels of activin A were reported to be correlated with cardiovascular diseases such as pulmonary hypertension, ges- tational hypertension, and atherosclerosis, and were related to disease severity and clinical outcomes. ${ }^{6,8-10,12}$ Our recent study also showed that the serum activin A level was associated with SBP and PP. ${ }^{13}$ In addition, Yndestad et al showed that patients with HFrEF have enhanced serum levels of activin A, associated with functional, hemodynamic, and neurohormonal parameters of disease severity. ${ }^{8}$ Elevated serum activin A was also reported to be associated with death and LV remodeling after myocardial infarction. ${ }^{21}$ These reports are in line with our finding that elevated circulating activin A concentrations not only correlated with echocardiographic DD with significantly enhanced LVMI, LAD, and RSVP, but also associated with a higher serum titer of NT-proBNP and prevalence of HFpEF at study enrollment. These results extend our current knowledge of DD and suggested a crucial pathophysiological role of activin A in the development of DD and resultant HF.

The underlying mechanism of DD remains elusive. Cardiac fibrosis is essential for the development of DD and progression to HF. As the myocardium is composed largely of cardiac fibroblasts, their differentiation into myofibroblasts and subsequent proliferation, synthesis, and release of extracellular collagens I and III are the main elements in cardiac fibrosis. ${ }^{3}$ A recent study suggested that activin A plays a critical role in cardiac fibrosis. It was shown to promote cardiac fibroblast proliferation and differentiation into myofibroblasts, with partial mediation of these effects through the extracellular signal-regulated kinases $1 / 2$ (ERK1/2) and p38 mitogen-activated protein kinase (p38 MAPK) pathways. ${ }^{22}$ These data suggest that activin $\mathrm{A}$ is a potential therapeutic target for cardiac fibrosis.

Although cell-line studies had suggested that activin $A$ is associated with cardiac fibrosis, evidence connecting bench studies to clinical DD is uncertain. We conducted this study to investigate the relationship between activin A and clinical DD. We observed higher serum levels of activin A to be associated with advanced age and higher prevalence of the underlying hypertensive disorder, chronic kidney disease, and HFpEF. Echocardiographic parameters, including the medial E/e' value, LAD, RVSP, and LVMI, increased significantly with the activin A level. Activin A concentrations remained significantly associated with DD and the composite endpoint, including death and new-onset $\mathrm{HF}$, even after adjusting age, BP, eGFR, smoking and antihypertensive medication. Moreover, the hs-CRP and NT-pro BNP levels positively correlated to the activin A levels, which suggests that chronic inflammation exists in patients with high activin A levels and may cause DD and HFpEF in turn.

\section{Study Limitations}

First, our study only revealed a correlation between DD and higher levels of activin A. Although previous cell and animal studies have suggested that activin $\mathrm{A}$ is a key factor in cardiac fibrosis, direct evidence for a causal relationship between activin A and DD is still lacking. Second, cardiac fibrosis is just 1 mechanism of DD. Some confounding factors such as obesity, smoking, and menopause could have contributed to DD in this study. Though we adjusted the inference of BMI and smoking, we could not confirm whether enrolled female patients were menopausal or not because of the retrospective study design. Third, serum levels of other inflammatory cytokines that could further identify the role of activin $\mathrm{A}$ in cardiac fibrosis, such as TGF- $\beta$, were not measured. Fourth, the accuracy of echo- 
cardiographic diagnostic criteria for DD remains controversial and is modified by times and different cardiology groups. Limited by the starting time of our program (2010) and the measurements the echocardiography laboratory could provide, we used an older version of echocardiographic diagnostic criteria. Some bias might exist, especially for patients with AF. The absence or a trivial grade of tricuspid regurgitation may cause an inaccurate estimate of RVSP and grading of the severity of DD. But this effect would be minimized by adopting the older version of echocardiographic diagnostic criteria. Moreover, the total enrolled case numbers were relatively small. Even though the association between higher activin A and HFpEF at enrollment was statistically significant, a larger population might be required to confirm the association. Finally, our study population consisted of people who had angina symptoms, which may induce selection bias, as these patients are more willing to undergo serial examinations and tend to have more cardiovascular disease, including obstructive coronary artery disease, which may superimpose the incidence of DD.

\section{Conclusions}

In conclusion, this study revealed an association between the serum level of activin A and cardiac DD. The results contribute to further understanding of characteristics shared between DD and HFpEF. Activin A is a potential biomarker for progression of DD to symptomatic HF.

\section{Acknowledgments}

This study was supported, in part, by research grants from the Ministry of Science and Technology of Taiwan (MOST 104-2314-B-075-047), the Novel Bioengineering and Technological Approaches to Solve Two Major Health Problems in Taiwan sponsored by the Taiwan Ministry of Science and Technology Academic Excellence Program (MOST 106-2633-B-009-001), the Ministry of Health and Welfare (MOHW106-TDU-B-211-113001), and Taipei Veterans General Hospital (V105C-0207, V106C-045). These funding agencies had no influence on the study design, data collection or analysis, decision to publish, or preparation of the manuscript.

\section{Conflict of Interest}

All authors declare no conflicts of interest.

\section{References}

1. Redfield MM, Jacobsen SJ, Burnett JC Jr, Mahoney DW, Bailey KR, Rodeheffer RJ. Burden of systolic and diastolic ventricular dysfunction in the community: Appreciating the scope of the heart failure epidemic. JAMA 2003; 289: 194-202.

2. Kane GC, Karon BL, Mahoney DW, Redfield MM, Roger VL, Burnett JC Jr, et al. Progression of left ventricular diastolic dysfunction and risk of heart failure. JAMA 2011; 306: 856-863.

3. Jeong EM, Dudley SC Jr. Diastolic dysfunction. Circ J 2015; 79: $470-477$.

4. Reed AL, Tanaka A, Sorescu D, Liu H, Jeong EM, Sturdy M, et al. Diastolic dysfunction is associated with cardiac fibrosis in the senescence-accelerated mouse. Am J Physiol Heart Circ Physiol 2011; 301: H824-H831.

5. Hedger MP, de Kretser DM. The activins and their binding protein, follistatin-Diagnostic and therapeutic targets in inflammatory disease and fibrosis. Cytokine Growth Factor Rev 2013;
24: $285-295$.

6. Engelse MA, Neele JM, van Achterberg TA, van Aken BE, van Schaik RH, Pannekoek H, et al. Human activin-A is expressed in the atherosclerotic lesion and promotes the contractile phenotype of smooth muscle cells. Circ Res 1999; 85: 931-939.

7. Michel U, Ebert S, Phillips D, Nau R. Serum concentrations of activin and follistatin are elevated and run in parallel in patients with septicemia. Eur J Endocrinol 2003; 148: 559-564.

8. Yndestad A, Ueland T, Oie E, Florholmen G, Halvorsen B, Attramadal H, et al. Elevated levels of activin A in heart failure: Potential role in myocardial remodeling. Circulation 2004; 109: $1379-1385$.

9. Miyoshi T, Hirohata S, Uesugi T, Hirota M, Ohnishi H, Nogami $\mathrm{K}$, et al. Relationship between activin A level and infarct size in patients with acute myocardial infarction undergoing successful primary coronary intervention. Clin Chim Acta 2009; 401: 3-7.

10. Yndestad A, Larsen KO, Oie E, Ueland T, Smith C, Halvorsen $\mathrm{B}$, et al. Elevated levels of activin A in clinical and experimental pulmonary hypertension. J Appl Physiol 2009; 106: 1356-1364.

11. Fukushima N, Matsuura K, Akazawa H, Honda A, Nagai T, Takahashi T, et al. A crucial role of activin A-mediated growth hormone suppression in mouse and human heart failure. PLoS One 2011; 6: e27901.

12. Yong HE, Murthi P, Wong MH, Kalionis B, Cartwright JE, Brennecke SP, et al. Effects of normal and high circulating concentrations of activin A on vascular endothelial cell functions and vasoactive factor production. Pregnancy Hypertens 2015; 5: $346-353$.

13. Tsai YL, Chang CC, Liu LK, Huang PH, Chen LK, Lin SJ. The Association between serum activin a levels and hypertension in the elderly: A cross-sectional analysis from I-Lan Longitudinal Aging Study. Am J Hypertens 2018; 31: 369-374.

14. Levey AS, Stevens LA, Schmid CH, Zhang YL, Castro AF 3rd, Feldman HI, et al. A new equation to estimate glomerular filtration rate. Ann Intern Med 2009; 150: 604-612.

15. Wachtell K, Bella JN, Liebson PR, Gerdts E, Dahlof B, Aalto T, et al. Impact of different partition values on prevalences of left ventricular hypertrophy and concentric geometry in a large hypertensive population: The LIFE study. Hypertension 2000; 35: $6-12$.

16. Roman MJ, Pickering TG, Schwartz JE, Pini R, Devereux RB. Relation of arterial structure and function to left ventricular geometric patterns in hypertensive adults. $\mathrm{J} \mathrm{Am} \mathrm{Coll} \mathrm{Cardiol}$ 1996; 28: 751-756.

17. Nagueh SF, Appleton CP, Gillebert TC, Marino PN, Oh JK, Smiseth OA, et al. Recommendations for the evaluation of left ventricular diastolic function by echocardiography. Eur J Echocardiogr 2009; 10: 165-193.

18. Okamoto Y, Fujita S, Morita H, Kizawa S, Ito T, Sakane K, et al. Association between circulating FGF23, alpha-Klotho, and left ventricular diastolic dysfunction among patients with preserved ejection fraction. Heart Vessels 2016; 31: 66-73.

19. Kusunose K, Yamada H, Nishio S, Tomita N, Niki T, Yamaguchi $\mathrm{K}$, et al. Clinical utility of single-beat E/e' obtained by simultaneous recording of flow and tissue Doppler velocities in atrial fibrillation with preserved systolic function. JACC Cardiovasc Imaging 2009; 2: 1147-1156.

20. Yancy CW, Jessup M, Bozkurt B, Butler J, Casey DE, Drazner MH, et al. 2013 ACCF/AHA Guideline for the management of heart failure: Executive summary: A report of the American College of Cardiology Foundation/American Heart Association Task Force on Practice Guidelines. J Am Coll Cardiol 2013; 62: $1495-1539$.

21. Lin JF, Hsu SY, Teng MS, Wu S, Hsieh CA, Jang SJ, et al. Activin A predicts left ventricular remodeling and mortality in patients with ST-elevation myocardial infarction. Acta Cardiol Sin 2016; 32: 420-427.

22. Hu J, Wang X, Wei SM, Tang YH, Zhou Q, Huang CX. Activin A stimulates the proliferation and differentiation of cardiac fibroblasts via the ERK1/2 and p38-MAPK pathways. Eur $J$ Pharmacol 2016; 789: 319-327. 